\title{
Measuring inline velocity profile of shampoo by electrical resistance tomography (ERT)
}

DOI:

10.1016/j.flowmeasinst.2017.09.013

\section{Document Version}

Accepted author manuscript

Link to publication record in Manchester Research Explorer

\section{Citation for published version (APA):}

Ren, Z., Kowalski, A., \& Rodgers, T. (2017). Measuring inline velocity profile of shampoo by electrical resistance tomography (ERT). Flow Measurement and Instrumentation, 58, 31-37.

https://doi.org/10.1016/j.flowmeasinst.2017.09.013

\section{Published in:}

Flow Measurement and Instrumentation

\section{Citing this paper}

Please note that where the full-text provided on Manchester Research Explorer is the Author Accepted Manuscript or Proof version this may differ from the final Published version. If citing, it is advised that you check and use the publisher's definitive version.

\section{General rights}

Copyright and moral rights for the publications made accessible in the Research Explorer are retained by the authors and/or other copyright owners and it is a condition of accessing publications that users recognise and abide by the legal requirements associated with these rights.

\section{Takedown policy}

If you believe that this document breaches copyright please refer to the University of Manchester's Takedown Procedures [http://man.ac.uk/04Y6Bo] or contact uml.scholarlycommunications@manchester.ac.uk providing relevant details, so we can investigate your claim.

\section{OPEN ACCESS}




\title{
Measuring inline velocity profile of shampoo by electrical resistance tomography (ERT)
}

\author{
Z. Ren ${ }^{\mathrm{a}, *}$, A. Kowalski ${ }^{\mathrm{b}}$, T.L. Rodgers ${ }^{\mathrm{a}}$ \\ ${ }^{a}$ School of Chemical Engineering and Analytical Science, University of Manchester, Oxford \\ Road, Manchester, M13 9PL, U.K. \\ ${ }^{b}$ Unilever R\&D, Port Sunlight Laboratory, Quarry Road East, Bebington, Wirral, CH63 \\ 3JW, U.K.
}

\begin{abstract}
The rheological properties of fluid systems govern the efficiency and quality of a production process. For certain materials, i.e. non-Newtonian fluids, The change in shear rate leads to the change of local viscosity, while the conductivity also changes due to the change in micro-structure of the material by varying shear rate. This paper provides a method of measuring the local velocity of shampoo in an in-line pipeline loop using electrical resistance tomography (ERT). Boundary voltages are measured by ITS P2000 ERT system and processed into a 2-D conductivity distribution in a MATLAB based ERT tool kit - EIDORS. Linear-back projection (LBP) method is used for solving the inverse problem. The change in shear rate leads to a micro-structure change in the shampoo, resulting in an annular conductivity distribution in the crosssectional view of the pipeline. By varying the flow rate, different changes in conductivity can be obtained. A velocity profile of shampoo is calculated based on the change in conductivity, giving a method to realise in-line rheometer by ERT.

Keywords: ERT, local velocity, micro-structure, Shampoo
\end{abstract}

*Corresponding authour. Email: zhen.ren@manchester.ac.uk

Preprint submitted to Flow measurement and Instrumentation 


\section{Introduction}

The rheological properties of fluid systems govern the efficiency and quality of a production process. Traditional method is to transfer off-line rheological properties by off-line rheometers to a real process flow, with unsatisfactory re5 sults in most cases. The reason of this failure is that an off-line analysis can only provide a retrospective snapshot of the sample rheology, which may not reflect the sample behaviour of the flow sample, particular for non-Newtonian flow, before or after the sampling time. As most fluids in chemical industry are non-Newtonian, an increasing demand has been placed for an in-line rheometer to obtain in-line and real-time rheological flow properties.

Many techniques were investigated to visualise flow in the process line. Some commercialised process rheometers, e.g. Brookfield and TA Instrument Rheometers, are commonly used in laboratory with limited success, because these rheometers use the same techniques as traditional off-line rheometers those have a low rate of data acquisition. New techniques were suggested in many literatures, including ultrasound Doppler velocity profiling techniques (UVP) [1, 2, laser Doppler anemometry measurements of velocity profile in combination with pressure difference (LDA-PD) [3, 4, optical tomography [5], nuclearbased imaging techniques and viscometers based on nuclear magnetic resonance

${ }_{20}$ (NMR) [6, 7. However, limitations by using the above techniques are observed. The optical tomography and the laser techniques are limited to transparent and dilute system [1]. The rheometer by nuclear and NMR technique is seldom used in actual process lines due to the safety and economical issues. Ultrasound is the most popular technique, but is restricted to be used when adding particles as the tracer or when measuring velocity of dispersed phase in a two-phase flow system [8, 9]. Ultrasound technique is also heavily affected by any air within the system, thus limited in applications [10].

In recent decades, electrical tomography was investigated for many industrial processes as it is a non-intrusive technique and has many advantages over other tomography techniques, e.g. low cost, no radiation, easy implementation and 
high speed. The fundamental of electrical tomography is to image an electrical property distribution within an object (vessel) by measuring electrical properties from a series of electrodes mounted on the surface of the object (vessel). The electrical property can be resistance/conductance, known as electrical resistance tomography (ERT) for measuring conductive material, or capacitance, known as electrical capacitance tomography (ECT), which is suitable for an image of dielectric materials [11. ERT is more suitable to visualise shampoo due to it being conductive, the conductivity of which is more than 100 times of tap water.

Common method for measuring the velocity profile is to add a tracer into the two or more sensors can be calculated by a cross-correlation method and the velocity profile is then obtained as the distance between sensors is known. The cross-correlation based method has been published with some success in obtaining velocity profile for Newtonian and non-Newtonian fluids [12, 13, 14]. Gen-

45 erally, the tracers are of three types: (1) nuclear-based tracer, such as positron emission or radioactive particles, which is used for nuclear based rheometer (2) optical particle for optical tomography, (3) other particles, which are used to make a contrast to the sample that can be detected by sensors, e.g. air bubbles, metal and plastic beads [10. However, the challenge of adding the tracer is that the cross-sectional position of the tracer always changes due to the density of the tracer, resulting in inaccurate localised information. In practical situation, it is undesirable to add any tracer to the product fluid in an industrial process line.

To avoid the drawbacks from adding a tracer, the authors [15 introduced an idea of estimating local velocity of shampoo in a pipeline using ERT. This paper details the method and compares it to theoretical models. This method is based on the fact that the conductivity varies with the micro-structure change due to the local shear rate. The viscosity and conductivity response to shear rate is investigated by a tomography rheometer system. 


\section{Shampoo behaviour: viscosity and conductivity response to shear rate}

As a common industrial product, shampoo is a cleaning agent for personal care, in particular for hair care. The primary ingredients of shampoo include water, surfactants, thickeners, conditioning agents, oil and special additives. The recipe of shampoo is relevant to many factors that make the shampoo behaviour much more complicated than any Newtonian material, e.g. water and oil. Generally, a formulated shampoo shows shear thinning behaviour that its viscosity is not just a function of temperature, but of the shear rate, i.e. at a fixed temperature, the viscosity of a shear thinning material decreases as the shear rate increases. As the shear rate differs in the pressure driven flow, this change in shear rate would result in the variance of the local viscosity and conductivity.

The viscosity and conductivity response of the shampoo to shear rate can be analysed by shearing the sample in a tomography rheometer. The tomography rheometer, as shown in Figure 1. is in a 'cup and bob' structure, measuring the torque corresponding to a selected rotational speed applied to the bob. For tomography purpose, the rheometer is made of plastic. The flow produced by the rotating bob in the cup is small, remaining Couette flow [16]. As the conductivity of the shampoo is temperature dependent, it is essential to eliminate the effect from any temperature change. The tomography rheometer is completed by a heating jacket, so that the temperature of the sample flow can be controlled and remained by water bath. The temperature can be monitored by a thermometer probe (Pico PT100) having a resolution of $0.1^{\circ} \mathrm{C}$. In the experiment, temperature was hold at $21^{\circ} \mathrm{C}$. The conductivity of shampoo sample is ${ }_{85} \quad 2.7 \mathrm{~S} \mathrm{~m}^{-1}$ at this temperature without shearing.

The central tube (cup) has an inner diameter of $4.2 \mathrm{~cm}$ and two planes of sixteen $0.2 \mathrm{~cm}$ in diameter circular electrodes towards the bottom, which allow the use of ERT. The agitator (bob) is concentric to the cup with a diameter of $3.7 \mathrm{~cm}$, therefore there is a gap of approx. $0.25 \mathrm{~cm}$ in between the cup and bob, 


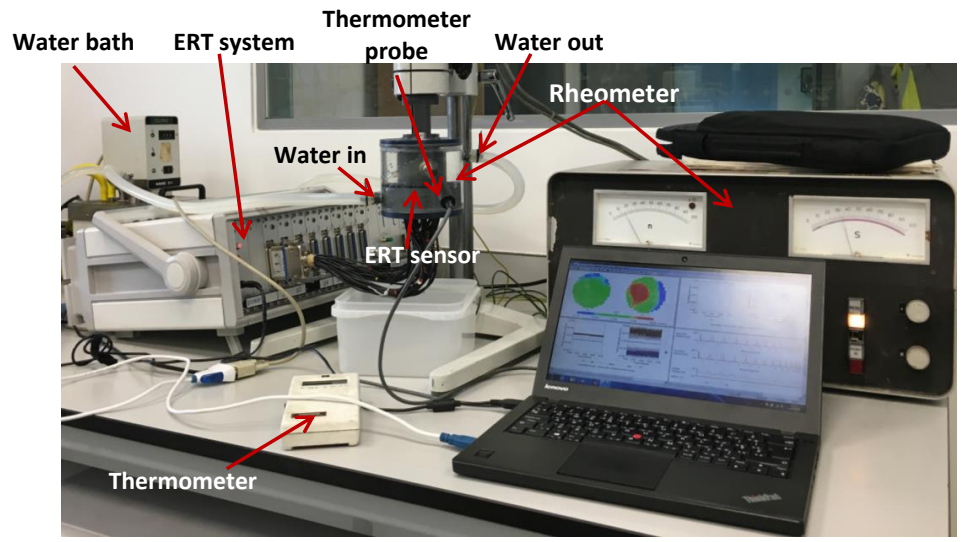

a

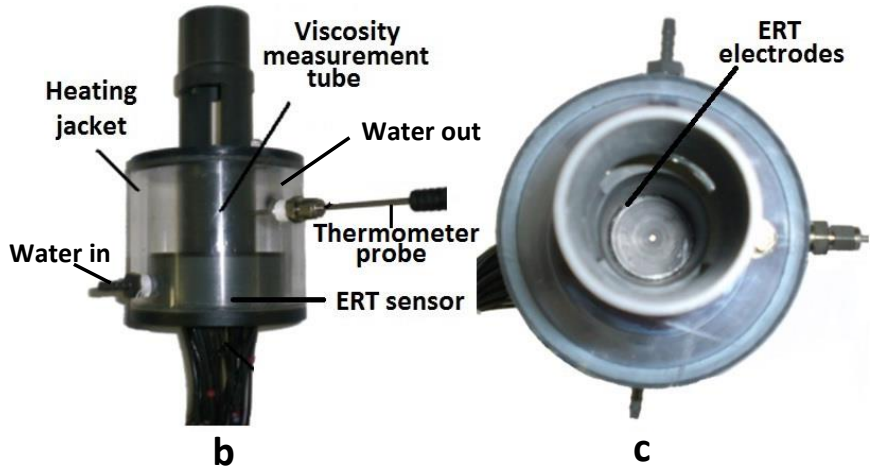

Figure 1: Tomography rheometer in cup and bob structure: (a) overview, (b) side view, (c) top view

which allows about $60 \mathrm{ml}$ of sample to be tested in the tomography rheometer. ITS P2000 ERT system was employed for measuring the boundary voltages between electrode pairs. The data acquisition setting is detailed in Table 1. Due to the size of the agitator, only the voltages between adjacent electrode pairs were measured. The relative change in conductivity $\left(\% \sigma_{r}\right)$ is directly calculated by the measured voltages,

$$
\% \sigma_{r}=\frac{\frac{I}{V_{m}}-\frac{I}{V_{0}}}{\frac{I}{V_{0}}}=\left(\frac{V_{0}}{V_{m}}-1\right) \times 100
$$

where $I$ is the injected current, which is a constant, $V_{m}$ and $V_{0}$ are the mean voltages of all adjacent pairs with and without shearing. The shear rate is set 
to increase from $0.9 \mathrm{~s}^{-1}$ to $57.6 \mathrm{~s}^{-1}$ and decrease back down to $0.9 \mathrm{~s}^{-1}$. Conductivity of shampoo at each shear rate is calculated from a repeat of 250 frames of voltage measurements. An interval of 2 minutes was taken for every 50 frames. Ten-minute interval was taken as changing to the next shear rate, to make sure no transient change in the viscosity, e.g. relaxation.

\begin{tabular}{cc}
\multicolumn{2}{c}{ Table 1: ITS P2000 Data Acquisition Settings } \\
\hline \hline Setting & Value \\
\hline Excitation frequency & $9.6 \mathrm{kHz}$ \\
Sampling time interval (between frames) & $0 \mathrm{~ms}$ \\
Samples per frame & 1 \\
Frames per download & 1 \\
Inject current & $75 \mathrm{~mA}$ \\
Sampling strategy & Adjacent \\
Delay cycles & 5 \\
\hline
\end{tabular}

The change in shear rate governs the viscosity change, meanwhile, conductivity changes due to the change in micro-structure of the sample by varying the shear rate. Shampoo is made up of elongated micelles. With the increase of the shear rate, these micelles start to align, this means that it is easier for electrons to flow past the micelles. As the shear rate continuously increases, these micelles become fully aligned, making the viscosity reduces as the micelles can easily move past each other (they move past each other at a constant shear stress), this allows the electron to move more freely, thus increasing the conductivity. At a very high shear rate, ions are broken free from the micelles, increasing the conductivity more. Figure 2 shows the dynamic viscosity and relative change in conductivity of shampoo corresponding to the shear rate. The red circular points are the viscosity/conductivity response to the increas105 ing shear rate, whilst the blue diamond points are the response to the decreasing shear rate. Significant decrease of viscosity occurs when the shear rate reaches $7.2 \mathrm{~s}^{-1}$ whilst the conductivity of shampoo increases with the increase in the 
shear rate. By increasing shear rate from $0.9 \mathrm{~s}^{-1}$ to $57.6 \mathrm{~s}^{-1}$, the viscosity decreases by approx. $70 \%$ and the relative change in conductivity increases by $1 \%$. However, when the shear rate decreases back to $0.9 \mathrm{~s}^{-1}$, the viscosity is $30 \%$ higher than the sample without shearing and the conductivity decreases by about $0.4 \%$. Two possible reasons for this difference in conductivity are (1) the defects in shampoo structure caused by increasing shear rate and (2) the very thin boundary layer of material forming at the outside of the viscometer, which does not influence the bulk viscosity, but leads to an electrically low resistance path for the charge carrying particles to flow through.
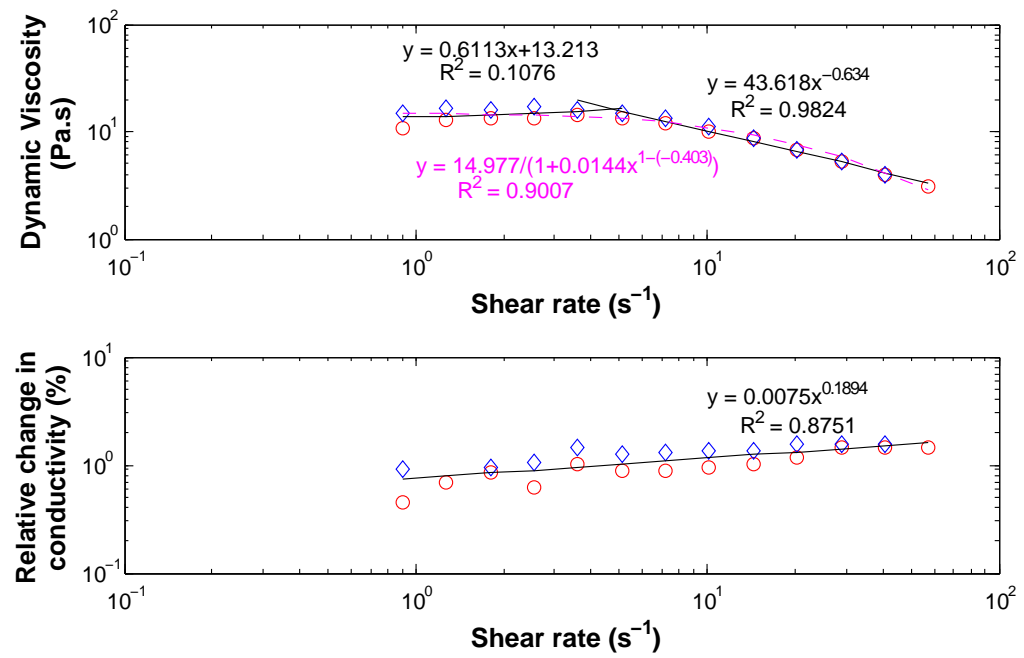

Figure 2: Shampoo's viscosity and conductivity response to shear rate

\section{Electrical Resistance Tomography (ERT)}

The ERT sensor mounted on the pipeline loop consists of 6 planes of 16 electrodes. The adjacent measurement strategy was used, which is to inject current between two adjacent electrodes and measure the voltage of all other two adjacent electrode pairs. Thus, 104 independent voltage measurements were 
taken for each plane and 624 measurements in total. The signal-to-noise ratio (SNR) of the ERT system is calculated by,

$$
S N R=10 \log _{10} \frac{\sum_{i=1}^{n} v_{i}^{2}}{\sum_{i=1}^{n}\left(v_{i}-\bar{v}\right)^{2}}
$$

where $v_{i}$ and $\bar{v}$, are the measured voltages of $i^{t h}$ frame and the mean voltages of $n$ frames, $n=50$. Note that $S N R$ is a vector which contains 624 elements, responding to the SNR of each channel. By averaging the 624 elements, the mean SNR of the ERT system with the sensor full of shampoo is $59.8 \mathrm{~dB}$.

Two basic problems need to be solved for an ERT system. One is forward problem, which is to calculate electrical field between electrode pairs with a known sensor structure and generate a Jacobian matrix of sensitivity distribution. The other is inverse problem, in which the conductivity distribution is determined from the measured boundary voltage data.

To calculate a sensitivity matrix for a 2-D ERT system, an electrostatic field can be assumed as the excitation frequency is low. According to the Maxwell's equations, the internal electric charge of the electrostatic field is zero, which gives

$$
\nabla(\sigma(x, y) \nabla \phi(x, y))=0
$$

where is $\sigma(x, y)$ the conductivity distribution and $\phi(x, y)$ is the electrical potential distribution.

Many numerical methods are used for solving the partial differential equations in forward problem, such as finite element method (FEM), boundary element method (BEM) and finite difference method (FDM). In this paper, the forward problem has been solved by a MATLAB based ERT toolkit-Electrical Impedance Tomography and Diffusion Optical Tomography Reconstruction Software (EIDORS). The mathematical theory behind the function in the software was introduced in [17, 18]. EIDORS uses FEM, which is to mesh the sensing area domain $\Omega$ into numbers of non-uniform non-overlapping elements (normally in triangular shape for the 2 -D modelling). The sensitivity in $\Omega$ can be written 
as a function of electrical field.

$$
S_{i, j}=\sum_{k=1}^{K} \sigma_{k} \iint_{\Omega_{k}} \nabla \phi_{i} \cdot \nabla \phi_{j} d x d y
$$

where $\sigma_{k}$ is the conductivity of element $k$. For generating a sensitivity matrix, a homogeneous conductivity distribution is used, i.e. $\sigma_{k}$ is set to a constant within the domain. $\phi_{i}$ and $\phi_{j}$ are the potential distribution when inject current at electrode pair $i$ and $j, K$ is the total number of elements in the domain.

Many reconstruction methods were undertaken for solving the ill-posed inverse problem of ERT. The most commonly used method in industry is Linearback projection (LBP), which is compromised between imaging speed and image quality. The conductivity distribution, $g$, in sensing domain is calculated by a linearised relationship between the boundary voltage, associated with conductance, and sensitivity matrix $S$ calculated by Eq. (4),

$$
g=S^{-1} \lambda
$$

where $\lambda$ is the change in boundary voltage. Unfortunately, the inverse of $\mathrm{S}$ does not exist because it is a non-square matrix. If $S$ is considered as a linear mapping from the conductivity vector space to the boundary voltage space, $S^{T}$ can be considered as a related mapping. An approximated solution $\hat{g}$ can be expressed as

$$
\hat{g}=\frac{S^{T} \lambda}{S^{T} \mu_{\lambda}}
$$

where $\mu_{\lambda}$ is an identity vector [19].

A 2-D model of 16 electrodes was built in MATLAB using EIDORS, the radius of which is $1.75 \mathrm{~cm}$. The image area was meshed into 538 triangular elements. The forward problem was solved by the four-electrode adjacent method and the sensitivity matrix is obtained. The images were reconstructed by Eq. (6) using differential data, which are the differences between the boundary voltages of the homogeneous phase (reference data) and the boundary voltages of the inhomogeneous phase (measurement data). 


\section{Estimation of the inline velocity profile based on ERT}

The idea of estimating velocity of shampoo by varying flow rate comes from the knowledge of the capillary rheometer, as shown in Figure 3 . According to Macosko 20, this rheometer is based on four assumptions: (1) the sample laminar flow should be fully developed, steady and isothermal; (2) there is no velocity along $r$ and $\theta$ direction; (3) there is no slip at the walls and (4) the fluid is incompressible with viscosity independent of pressure.

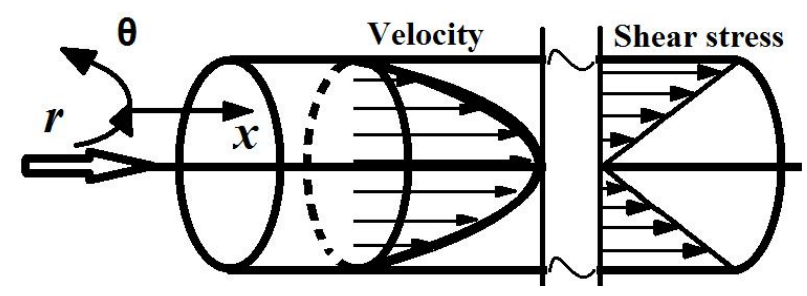

Figure 3: Sketch of velocity and shear stress in pipeline

An experiment was conducted with a recycled in-line process pipeline system, the piping and instrumentation diagram (P\&ID) is shown in Figure 4. The system consists of a 601 vessel with an agitator in the centre and a pump which circulates shampoo from the vessel around the system. The agitator allows mixing the shampoo in the vessel with the recycled shampoo. The pump was fully controlled by the control panel and the actual flow rate was read by a flow meter (F01) mounted on the pipeline system. A section of pipeline was replaced by the ERT sensor, placing horizontally. The inner radius of the ERT sensor is $1.75 \mathrm{~cm}$ and the distance between each ring is $5.3 \mathrm{~cm}$. Two out of four temperature probes were placed near the ERT sensor, one is at the inlet of the sensor (T02) and the other mounted with a conductivity meter (CS01) was placed at the outlet of the ERT sensor. Twenty flow rates were applied in the pipeline loop randomly, from $39 \mathrm{~kg} \mathrm{~h}^{-1}$ (5 rpm) to $928 \mathrm{~kg} \mathrm{~h}^{-1}$ (108 rpm). Voltage data were collected by ITS P2000 ERT system (parameter setting see Table 1) and processed in MATLAB for imaging. The data at flow rate of $10 \mathrm{~kg} \mathrm{~h}^{-1}(1.5 \mathrm{rpm})$ was used as the reference. 


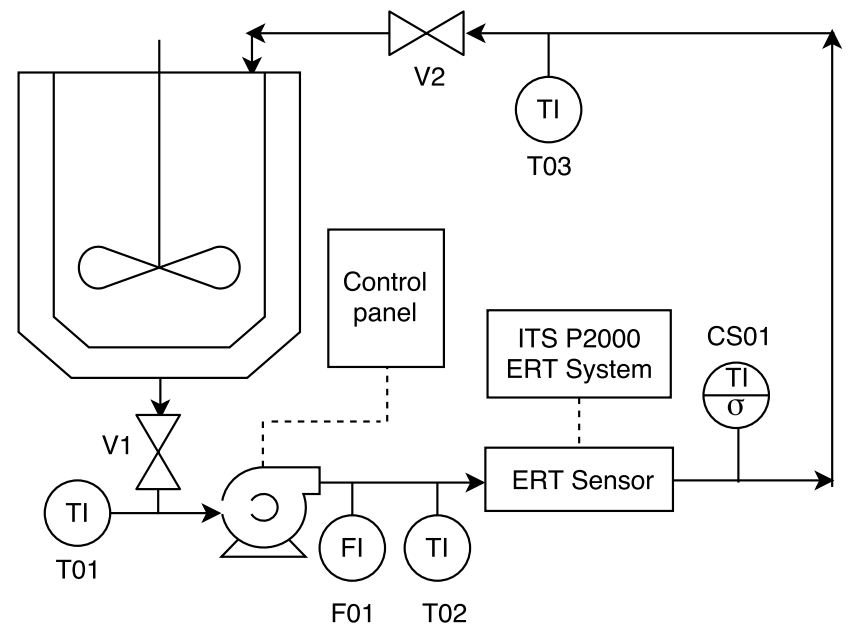

Figure 4: P\&ID of pipeline loop

\subsection{ERT images} results confirm the previous findings in the cup and bob rheometer that the conductivity varies by different shear rates, giving a possibility to estimate the local velocity of shampoo in the horizontal pipeline based on the conductivity distribution by ERT.

\subsection{Velocity fit}

As a result of previous findings from the study on the shampoo behaviour, the conductivity is a function of the shear rate. The velocity profile with the shear rate can be replaced by the velocity with the conductivity distribution in the pipeline. The conductivity data can be obtained by extracting the element data from the reconstructed images as shown in Figure 5. The conductivity of 


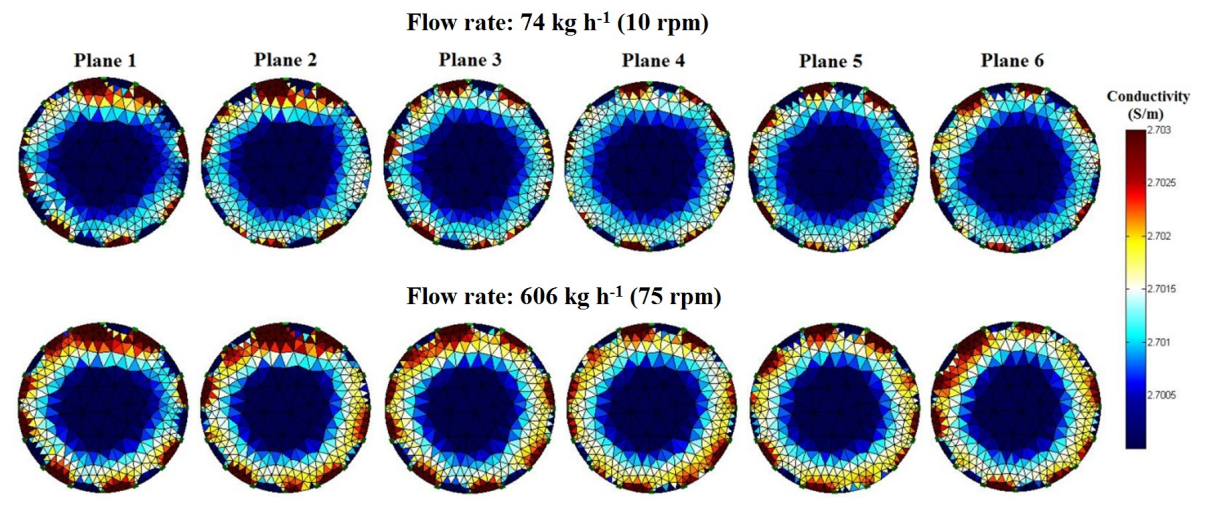

Figure 5: Reconstructed image at $74 \mathrm{~kg} \mathrm{~h}^{-1}$ and $606 \mathrm{~kg} \mathrm{~h}^{-1}$

each element is plotted against the related radius to the center of the pipeline, as shown in Figure 6 (a). The large variation of conductivities at the wall comes from the high sensitivity near electrodes which is sensitive to any noise in the reconstruction on the exterior elements. A four-order polynomial function $(f(r))$ was used to fit the local conductivity against its location (local radius). The red solid line in Figure 6(a) is the original fit for the conductivity and the green dashed line is the adjusted fitting line that to make sure all element data over the reference value $\left(f(r) \geq 2.7 \mathrm{~S} \mathrm{~m}^{-1}\right)$. If the local velocity is inversely linear to the change in shear rate, a representation of the shear rate, $\dot{\gamma}$, over the pipe can be written as,

$$
\dot{\gamma}=-\frac{d u}{d r}
$$

where $u$ is the local velocity, $r$ is the local radius of the element. Then $\dot{\gamma}$ is replaced by the function of conductivity to radius $(f(r))$ :

$$
u(r) \cong \int_{0}^{R}-f(r) d r
$$

where $u(r)$ is the velocity profile to radius, $\mathrm{R}$ is the inner radius of the pipeline, $R=1.75 \mathrm{~cm}$. According to the third assumption in the capillary rheometer, the velocity at the wall is $0(u(R)=0)$. A velocity profile can be calculated by Eq. 
(8) and plotted as shown in Figure 6(b). The calculated flow rate is given by

$$
Q_{c a l}=\int_{0}^{R} 2 \pi u(r) r d r
$$
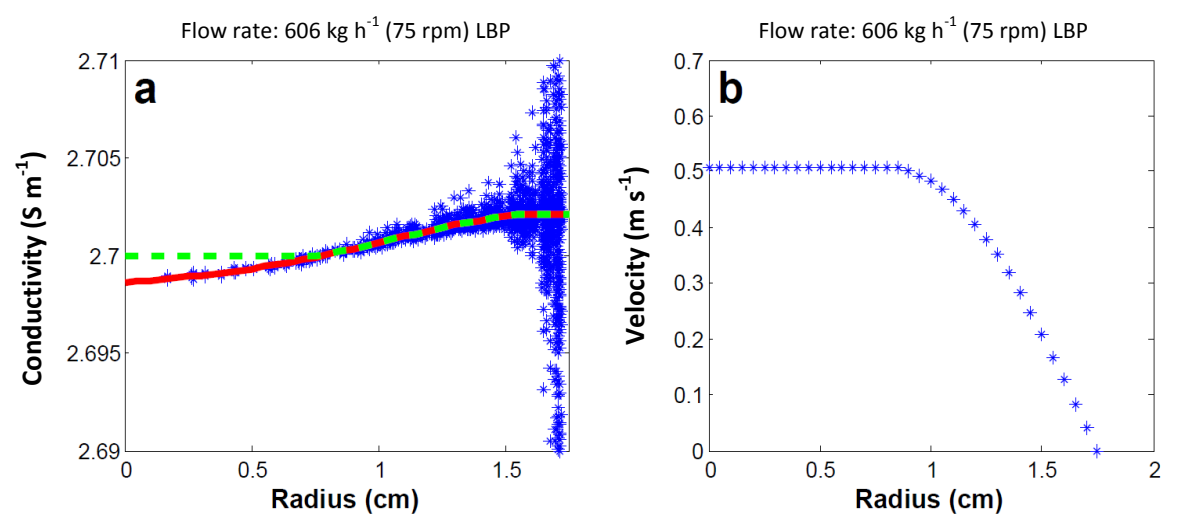

Figure 6: Velocity fit, (a) Local conductivity against local radius, (b) Calculated velocity against local radius

\subsection{Temperature fit}

The rheology of shampoo is highly temperature dependent. The viscosity of shampoo decreases by approx. $10 \%$ whilst the conductivity increases by $2.5 \%$ when the temperate increases by $1^{\circ} \mathrm{C}$. Analysis of the shampoo and its temperature response to flow rate was investigated by pumping the shampoo in the pipeline loop for 45 minutes at 4 flow rates, i.e. $111 \mathrm{~kg} \mathrm{~h}^{-1}, 223 \mathrm{~kg} \mathrm{~h}^{-1}$, $433 \mathrm{~kg} \mathrm{~h}^{-1}$ and $624 \mathrm{~kg} \mathrm{~h}^{-1}$. Figure 7 shows the change in calculated flow rate corresponding to the change in temperature. The higher the flow rate, the larger the change in temperature. The gradient $(\mathrm{m})$, regarding to the change in calculated ERT flow rate to the change in temperature, is plotted against the actual flow rate, as shown in Figure 7 (b). A trend line is plotted, using for the temperature fit to the velocity profile. The flow rate is calibrated to $25^{\circ} \mathrm{C}$, which can be expressed as

$$
Q_{\text {temp }}=Q_{\text {cal }} /\left(3123.5 \times Q_{\text {mea }}^{-0.793} \times\left(\frac{T}{25}-1\right)+1\right)
$$


where $Q_{\text {temp }}$ is the estimated flow rate after temperature fit, $T$ is the actual temperature, read from the temperature probe at the outlet of the ERT sensor and $Q_{m e a}$ is the actual flow rate, read from the flow meter, constants 3123.5 and -0.793 are calculated from fit of $\mathrm{m}$ to the actual flow rate, shown in Figure $7(b)$.
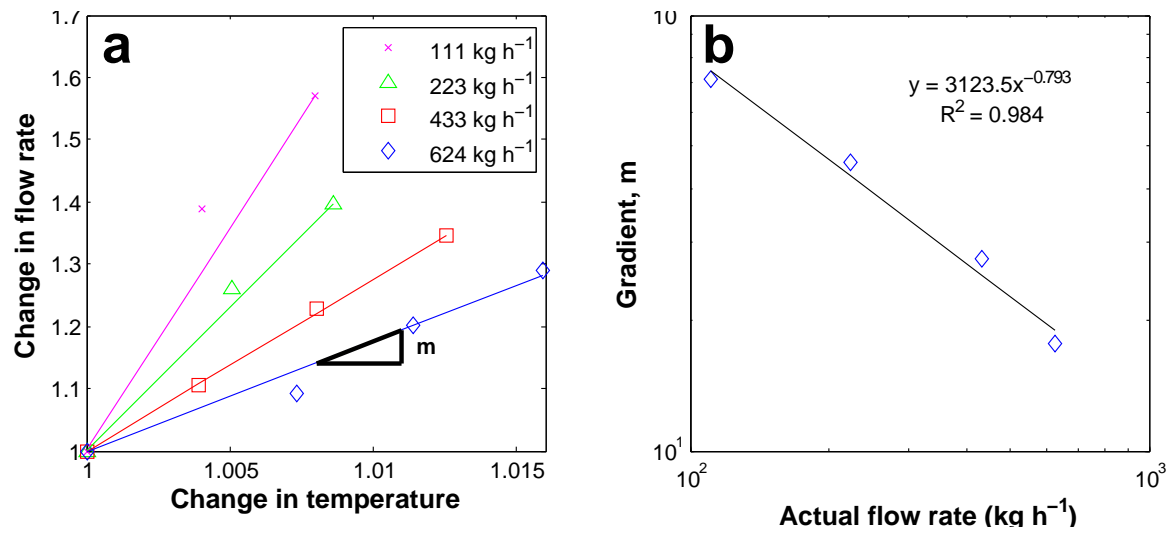

Figure 7: Temperature effect on flow rate by ERT, (a) Change in calculated flow rate by ERT against the change in temperature when pumping shampoo for 45 minutes, (b) the gradient of ERT flow rate change to temperature against actual flow rate

The scale between actual and temperature fitted flow rate is plotted against the actual flow rate and fitted by a linear line, as shown in Figure 8 . The line has a better fit to the low flow rates whilst more variation appears at high flow rates. The final flow rate is calibrated by a fixed scale factor to the actual flow rate.

Figure 9 plots the calibrated flow rate and the actual flow rate for the 20 measurements. Higher accuracy is achieved by ERT when pumping the shampoo at a lower flow rate. Notable difference between the actual and ERT flow rate is observed when continually pumping shampoo at high flow rate, which conduces to a large temperature change and causes the calibration of the flow rate by ERT out of the temperature fit. 


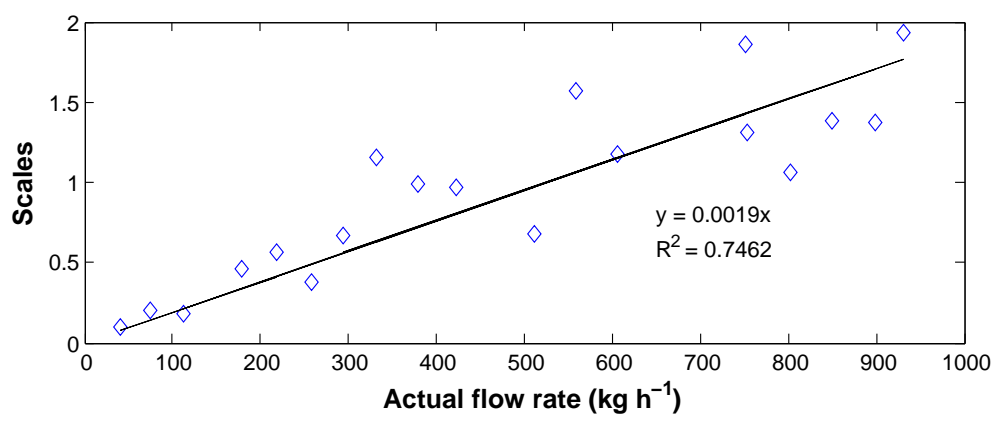

Figure 8: Calibration scales to actual flow rate

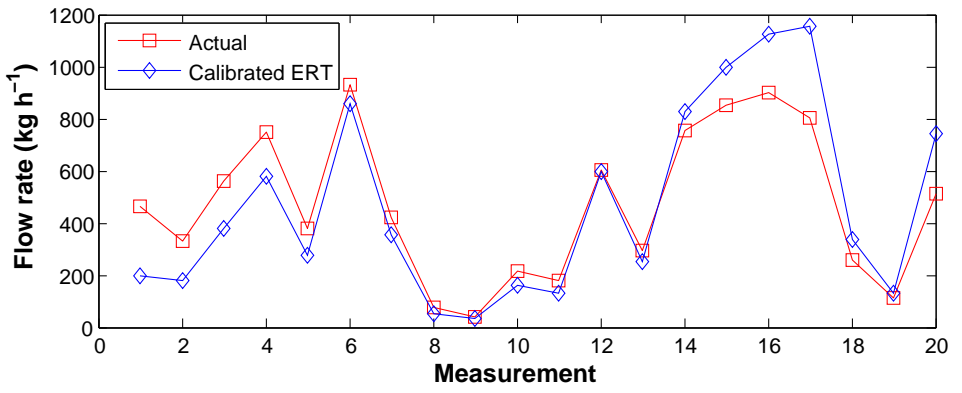

Figure 9: Comparison of calibrated ERT flow rate to actual flow rate

\subsection{Comparison to theoretical values}

Despite the viscosity profile fits well with Ellis model, as shown in Figure 2(a), the velocity profile is extremely difficult to derive. To simplify the calculation, shampoo viscosity is represented as a Newtonian model part and power-law model part. The shear stress $\tau$ within a circular pipe is represented by

$$
\tau=-\frac{r}{2}\left(\frac{\delta P}{\delta x}\right)
$$

where $\frac{\delta P}{\delta x}$ is the pressure drop gradient [20].

For the power-law model part of the profile, the shear stress is $n$ power of velocity gradient that can be given by

$$
\tau=K\left(\frac{\delta u}{\delta r}\right)^{n}
$$


where $K$ is a constant. Integrating into Eq. 111) produces a velocity profile of

$$
u=\left(-\frac{1}{2 K}\left(\frac{\delta P}{\delta x}\right)\right)^{1 / n}\left(\frac{n}{n+1}\right)\left(R^{(n+1) / n}-r^{(n+1) / n}\right)
$$

When using the no slip boundary condition, the shear thinning is at the highest shear rate, i.e. $u=0$ at $r=R$. The velocity profile can be calculated until the shear rate moves into the Newtonian model part of the viscosity curve, i.e. the lower shear rate at the centre of the pipe. In this model, the shear stress is simply proportional to the velocity gradient, which is given by

$$
\tau=\mu\left(\frac{\delta u}{\delta r}\right)
$$

where $\mu$ is a constant. Integrating into Eq. (11) produces a velocity profile of

$$
u=-\frac{1}{4 \mu}\left(\frac{\delta P}{\delta x}\right)\left(R_{C}^{2}-r^{2}\right)+u_{C}
$$

When using the boundary condition that the velocity is equal to the velocity calculated from the shear thinning part at the radius of change, i.e. $u=u_{C}$ at $r=R_{C}$. The velocity profile is integrated across the radius to calculate the total flow rate, the pressure drop is then calculated to match the total measured flow rate by the flow meter. Figure 10 plots the calibrated local velocity and the theoretical velocity profile by Eq. 13 and 15 at three flow rates, $218 \mathrm{~kg} \mathrm{~h}^{-1}$, $423 \mathrm{~kg} \mathrm{~h}^{-1}$ and $606 \mathrm{kgh}^{-1}$ by ERT. The velocity profiles by ERT are highly consistent with the theoretical values.
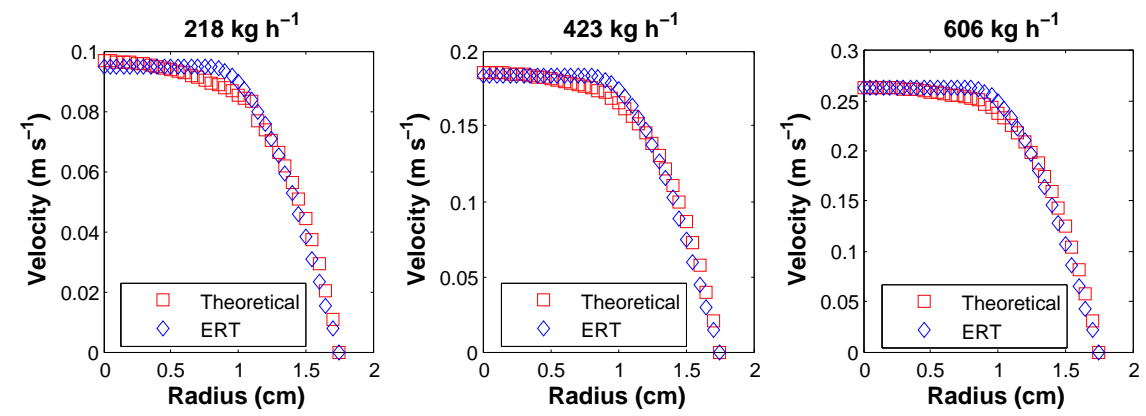

Figure 10: Theoretical and calibrated ERT velocity profile with radius 


\section{Conclusion}

205 the velocity profile based on the changes in conductivity due to the microstructure change of a material response to different shear. This effect could be used for a real-time in-line rheometer based on ERT. While many literatures have been published on the in-line rheometer based on several non-tomography process flow condition in laboratory. The method presented in this paper is implemented by calculating the velocity profiles of shampoo by varying flow rate only and has several advantages over those presented in previous papers. Firstly it is simple to implement as there is no tracer needed to be added. Then,

\section{Acknowledgement}

The authors appreciate the financial support from the U.K. Engineering and Physical Sciences Research Council (Grant EP/L505778/1) and Unilever.

\section{References}

225

[1] J. Wiklund, I. Shahram, M. Stading, Methodology for in-line rheology by ultrasound doppler velocity profiling and pressure difference techniques, 口

[ [2] T. Ihara, H. Kikura, Y. Takeda, Ultrasonic velocity profiler for very low 
133. doi:http://dx.doi.org/10.1016/j.flowmeasinst.2013.10.003.

[

URL http://www.sciencedirect.com/science/article/pii/ S0955598613001301

[3] J. Meissner, Polymer melt flow measurements by laser doppler velocime-

try, Polymer Testing 3 (4) (1983) 291-301. doi:10.1016/0142-9418(83) 90014-4.

[4] P. Brunn, T. Wunderlich, M. Muller, Ultrasonic rheological studies of a body lotion, Flow Measurement and Instrumentation 15 (3) (2004) 139144. doi:10.1016/j.flowmeasinst.2003.12.007.

[5] G. Faris, H. Hertz, Tunable differential interferometer for optical tomography, Applied Optics 28 (21) (1989) 4662-4667. doi:10.1364/A0.28. 004662

[6] K. Kose, Nmr imaging of turbulent structure in a transitional pipe flow, Journal of Physics D-Applied Physics 23 (7) (1990) 981-983. doi:10.1088/ $0022-3727 / 23 / 7 / 038$

[7] T. Li, J. Seymour, R. Powell, M. Mccarthy, K. Mccarthy, L. Odberg, Visualization of flow patterns of cellulose fiber suspensions by nmr imaging, Aiche Journal 40 (8) (1994) 1408-1411. doi:10.1002/aic.690400812.

¿ [8] C. Tan, Y. Yuan, X. Dong, F. Dong, Oil-water two-phase flow measurement with combined ultrasonic transducer and electrical sensors, Measurement Science and Technology 27 (12) (2016) 125307.

URL http: //stacks . iop.org/0957-0233/27/i=12/a=125307

[9] X. Dong, C. Tan, Y. Yuan, F. Dong, Measuring oil-water two-phase flow velocity with continuous-wave ultrasound doppler sensor and drift-flux model, IEEE Transactions on Instrumentation and Measurement 65 (5) (2016) 1098-1107. doi:10.1109/TIM.2015.2507740. 
[10] J. Chaouki, F. Larachi, M. Dudukovic, Noninvasive tomographic and velocimetric monitoring of multiphase flows, Industrial and Engineering Chemistry Research 36 (11) (1997) 4476-4503. doi:10.1021/ie970210t.

[11] T. York, Status of electrical tomography in industrial applications, J. Electronic Imaging 10 (3) (2001) 608-619. doi:10.1117/1.1377308.

[12] A. Sutherland, T. Long, E. Randall, A. Wilkinson, Determining concentration and velocity profiles of non-newtonian settling slurries using electrical resistance tomography, Journal of the South African Institute of Mining and Metallurgy 108 (10) (2008) 583-590. doi:10.1205/02638760152721398.

[13] C. Tan, F. Dong, Cross correlation velocity of oil-water two-phase flow by a dual-plane electrical resistance tomography system, in: 2010 IEEE Instrumentation Measurement Technology Conference Proceedings, 2010, pp. 766-770. doi:10.1109/IMTC.2010.5488276.

[14] M. Sharifi, B. Young, Electrical resistance tomography (ert) for flow and velocity profile measurement of a single phase liquid in a horizontal pipe, 口 Chemical Engineering Research and Design 91 (7) (2013) 1235-1244. doi: $10.1016 / \mathrm{j}$. cherd.2013.02.004.

[15] Z. Ren, A. Kowalski, T. L. Rodgers, Estimating inline velocity profile of shampoo by electrical resistance tomography (ert), in: 8th World Congress on Industrial Process Tomography, 2016, pp. 1-6.

URL http://www. isipt.org/world-congress/8/29094.html

[16] T. Rodgers, Multiple-scale approach to understanding formulated product production, Ph.D. thesis, the University of Manchester, Manchester, U.K. (2011).

[17] M. Vauhkonen, W. Lionheart, L. Heikkinen, P. Vauhkonen, J. Kaipio, A matlab package for the eidors project to reconstruct two-dimensional eit

【 images, Physiological Measurement 22 (1) (2001) 107-111. doi:10.1088/ $0967-3334 / 22 / 1 / 314$. 
[18] A. Adler, W. Lionheart, Uses and abuses of eidors: an extensible software base for eit, Physiological Measurement 27 (5) (2006) S25-S42. doi:10. 1088/0967-3334/27/5/S03.

[19] W. Yang, L. Peng, Image reconstruction algorithms for electrical capacitance tomography. measurement science and technology, Measurement Science and Technology 14 (1) (2003) R1-r13. doi:10.1088/0957-0233/14/ $1 / 201$.

[20] C. Macosko, RHEOLOGY: Principles, Measurements, and Applications, 1st Edition, VCH-Wesley, New York, 1994. 